centres studied. The data suggest capreomycin might be associated with less ototoxicity when compared to amikacin. There are no UK guidelines to inform best practice and better evidence, including clinical and cost-effectiveness studies, is needed to inform the implementation of current technology including genetic testing.

\section{S3 FACTORS FOR SUCCESSFUL TREATMENT COMPLETION AMONG MDR TB CASES IN THE UK}

doi:10.1136/thx.2010.150912.3

${ }^{1} \mathrm{~L}$ F A Anderson, ${ }^{1} \mathrm{~S} T$ Tamne, ${ }^{2} \mathrm{~J}$ W Watson, ${ }^{1} \mathrm{I}$ A Abubakar. ${ }^{1}$ Health Protection Agency, London, UK; ${ }^{2}$ Department of Respiratory Medicine, St James University Hospital, Leeds, UK

Background UK guidelines recommend at least 18 months treatment for patients with multi-drug resistant tuberculosis (MDR TB). Prior to 2008, treatment completion was only available for 12 months nationally in the UK, therefore the proportion completing treatment was unknown. There is also a lack of recent guidance for the treatment and management of MDR TB cases in resource rich low $\mathrm{TB}$ incidence settings.

Aims To report the treatment outcome at 24 months for MDR TB patients between 2004 and 2007 and examine treatment regimens and management associated with successful outcomes, taking clinical, social and demographic factors into account.

Methods A retrospective cohort study was used to follow-up MDR TB patients at 24 months. Patients were identified using the national surveillance system which contains demographic and clinical characteristics of cases and is matched routinely to reference laboratory data. Questionnaires were sent out to case managers to collect information on outcome of care and associated risk factors for treatment completion.

Results $69.6 \%(142 / 204)$ of patients completed treatment at 24 months and $2.9 \%$ (6) of these completed treatment within 12 months. $4.4 \%$ (9) were still on treatment, $6.9 \%$ (14) had their treatment stopped, $6.9 \%$ (14) died, $7.8 \%$ (16) were lost to follow-up and $4.4 \%$ (9) were transferred overseas. Only 40.3\% (77/191) patients started on treatment received directly observed therapy at any time. Treatment with a recommended fluoroquinolone $(\mathrm{OR}=2.3 ; 95 \% \mathrm{CI}$ 1.2 to $4.2, \mathrm{p}<0.000$ ) or a bacteriostatic (OR 2.86; 95\% CI 1.6 to 5.3 , $\mathrm{p}=0.001)$, a change in treatment regimen $(\mathrm{OR}=2.2 ; 95 \%$ CI 1.2 to 4.0 , $\mathrm{p}=0.01)$ and treatment with four or more effective drugs $(\mathrm{OR}=2.0$; $95 \%$ CI 1.1 to $3.8, p=0.02$ ) were significantly associated with a successful treatment outcome.

Conclusion The proportion of MDR TB cases completing treatment is similar to cases with drug susceptible disease due to the use of individualised treatment regimens. However, treatment completion still remains below World Health Organization targets.

\section{S4 EVALUATION OF TB PEER EDUCATORS-ESSENTIAL PARTNERS IN METROPOLITAN TB CONTROL}

doi:10.1136/thx.2010.150912.4

${ }^{1} \mathrm{~J}$ Hall, ${ }^{2} \mathrm{~S}$ Bethell, ${ }^{2} \mathrm{~S}$ Helleren, ${ }^{1} \mathrm{~A}$ Story, ${ }^{3} \mathrm{M}$ Lipman. ${ }^{1}$ Find \& Treat, Department of Health TB Projects, London, UK; ${ }^{2}$ Groundswell, London, UK; ${ }^{3}$ Royal Free Hampstead NHS Trust, London, UK

Background One in six of all notified tuberculosis (TB) cases in London are among homeless people, problem drug and alcohol users and prisoners (hard-to-reach). These groups are at high risk of delayed diagnosis, infectious and drug resistant TB and poor treatment outcomes. The Berlin Declaration (2007) stated that affected communities are essential partners in TB control. While initiatives involving hard-toreach communities in HIV control have proven effective, evidence to support their contribution to TB control activities is lacking. We aimed to improve service access and uptake of TB screening among hard-toreach groups by harnessing the authentic voice and experience of former TB patients from these affected communities.

Method Seven former TB patients with a history of homelessness and drug/alcohol dependence were recruited and trained as peer educators to work alongside TB clinics and a mobile x-ray screening service. Qualitative and quantitative methods were used to evaluate their impact on service access and screening uptake.

Results Peers recruited 3200 hard-to-reach clients at 101 screening sessions resulting in 45 hospital referrals between May 2009 and February 2010. Following TB peer training of homeless shelter hostel workers, screening uptake increased from $44 \%$ to $75 \%$. Subsequent structured interviews with service users highlighted the importance of peer educators in raising TB awareness and promoting service access. Conclusion Our evaluation demonstrated that trained peer educators can improve service access and TB screening uptake in the short and medium term in hard-to-reach groups. The success of this approach argues for greater peer educator involvement in strategies to control metropolitan TB.

Funding This project was supported by the Department of Health.

\section{S5 PREVALENCE OF LATENT TUBERCULOSIS INFECTION IN IMIMIGRANTS TO THE UK: FINDINGS FROM A MULTI- CENTRE STUDY}

doi:10.1136/thx.2010.150912.5

${ }^{1} \mathrm{M}$ Pareek, ${ }^{2} \mathrm{~J}$ P Watson, ${ }^{3} \mathrm{O} \mathrm{M}$ Kon, ${ }^{4} \mathrm{G}$ Woltmann, ${ }^{1} \mathrm{~A}$ Lalvani. ${ }^{1} / \mathrm{mperial}$ College London, London, UK; ${ }^{2}$ Leeds Teaching Hospital NHS Trust, Leeds, UK; ${ }^{3}$ Imperial College Healthcare NHS Trust, London, UK; ${ }^{4}$ University Hospitals Leicester NHS Trust, Leicester, UK

Background Notifications for tuberculosis (TB) in the United Kingdom (UK) increased by 30\% between 1992 and 2007 with overseas-born migrants accounting for $72 \%$ of cases in 2007 . Much of this increase is due to the synergy of migration from high-TB burden countries and the reactivation of pre-existing latent $\mathrm{TB}$ infection (LTBI). Since 2006, NICE guidance advocates screening for LTBI in all children from countries with a TB incidence $>40 / 100000$ and adults from Sub-Saharan Africa and countries with a TB incidence $>500$ / 100000 . The rationale underpinning these guidelines remains unclear particularly as there are little data on the prevalence of LTBI in immigrants to the UK from regions with different $\mathrm{TB}$ incidence rates. Aims To quantify the prevalence of LTBI in immigrants, assess factors associated with IGRA positivity and to determine LTBI yield from current screening thresholds recommended by NICE.

Methods Analysis of data prospectively obtained through IGRA testing (QuantiFERON-Gold/QuantiFERON-Gold-in-tube) of immigrants in three different centres in the UK during 2008-2010. Descriptive analyses were undertaken. Yields at different screening thresholds were calculated. Subsequent univariate and multivariate analyses were undertaken to assess independent factors associated with IGRA positivity; $p<0.05$ considered significant.

Results In total 915 immigrants were screened during the study period. Median age was 26.8 years (interquartile range $22-33$ ), $50.6 \%$ were female and $72.5 \%$ had previously been BCG vaccinated. $48.9 \%$ and $28.5 \%$ of migrants screened originated from the Indian Subcontinent and Sub-Saharan Africa, respectively. Overall 911/915 individuals had determinate IGRA results-199 (21.7\%) tested positive and $712(77.8 \%)$ tested negative; $4(0.4 \%)$ individuals had indeterminate results. Multivariate analysis revealed that increasing age $(p<0.0001)$ and increasing TB incidence of country of origin $(p=0.0014)$ were independently associated with IGRA positivity. Applying current NICE guidance resulted in a yield of 76/311 (24.4\%) but would miss $61.8 \%$ of the immigrant population with LTBI (Abstract S5 Table 1). 
Abstract S5 Table 1 Yields for latent TB infection (as defined by a positive QuantiFERON assay) for different age-groups and at different screening thresholds. Those in bold represent current NICE guidance (note: NICE also recommends screening those aged 16-35 from SubSaharan countries which is not shown on this table for clarity)

\begin{tabular}{|c|c|c|c|c|}
\hline $\begin{array}{l}\text { Age group and } \\
\text { TB incidence } \\
\text { screening } \\
\text { threshold (per 100000) }\end{array}$ & $\begin{array}{l}\text { Number } \\
\text { tested }\end{array}$ & $\begin{array}{l}\text { Number } \\
\text { positive }\end{array}$ & $\begin{array}{l}\text { Yield at incidence } \\
\text { level, that is proportion } \\
\text { of those tested giving a } \\
\text { positive result }(\%)\end{array}$ & $\begin{array}{l}\% \text { of all LTBI } \\
\text { identified if } \\
\text { threshold set } \\
\text { at this level }\end{array}$ \\
\hline \multicolumn{5}{|l|}{$<16$ years } \\
\hline Screen all & 85 & 5 & 5.9 & 100 \\
\hline Screen $\geq 40$ & 77 & 5 & 6.5 & 100 \\
\hline Screen $\geq 100$ & 76 & 4 & 5.3 & 80 \\
\hline Screen $\geq 200$ & 31 & 3 & 9.7 & 60 \\
\hline Screen $\geq 300$ & 11 & 2 & 18.2 & 40 \\
\hline Screen $\geq 400$ & 6 & 2 & 33.3 & 40 \\
\hline Screen $\geq 500$ & 6 & 2 & 33.3 & 40 \\
\hline \multicolumn{5}{|l|}{$16-35$ years } \\
\hline Screen all & 678 & 142 & 20.9 & 100 \\
\hline Screen $\geq 40$ & 666 & 142 & 21.3 & 100 \\
\hline Screen $\geq 100$ & 597 & 136 & 22.8 & 95.8 \\
\hline Screen $\geq 200$ & 314 & 69 & 21.9 & 48.6 \\
\hline Screen $\geq 300$ & 106 & 30 & 28.3 & 21.1 \\
\hline Screen $\geq 400$ & 41 & 8 & 19.5 & 5.6 \\
\hline Screen $\geq 500$ & 34 & 7 & 20.6 & 4.9 \\
\hline \multicolumn{5}{|l|}{ Over 35 years } \\
\hline Screen all & 147 & 52 & 35.4 & 100 \\
\hline Screen $\geq 40$ & 145 & 52 & 35.9 & 100 \\
\hline Screen $\geq 100$ & 113 & 44 & 38.9 & 84.6 \\
\hline Screen $\geq 200$ & 81 & 33 & 40.7 & 63.5 \\
\hline Screen $\geq 300$ & 25 & 14 & 56.0 & 26.9 \\
\hline Screen $\geq 400$ & 10 & 7 & 70.0 & 13.5 \\
\hline Screen $\geq 500$ & 10 & 7 & 70.0 & 13.5 \\
\hline
\end{tabular}

Conclusions Immigrants have a high prevalence of LTBI but current NICE guidance detects only $38.2 \%$ of new entrants with LTBI. Given the high rates of reactivation of LTBI in new entrants, our findings suggest that consideration should be given to reducing the screening threshold to include those from the Indian Subcontinent (incidence 170/100 000) which would result in $67.8 \%$ of all LTBI cases being identified.

\section{S6 SENSITIVITY AND SPECIFICITY OF MOBILE DIGITAL CHEST RADIOGRAPHY FOR THE DIAGNOSIS OF ACTIVE PULMONARY TUBERCULOSIS. A COHORT STUDY IN HIGH RISK GROUPS IN LONDON}

doi:10.1136/thx.2010.150912.6

${ }^{1} \mathrm{R}$ W Aldridge, ${ }^{2} \mathrm{~A}$ Story, ${ }^{2} \mathrm{H}$ Stagg, ${ }^{1} \mathrm{M}$ Lipman, ${ }^{3} \mathrm{~J}$ Knight, ${ }^{3} \mathrm{D}$ Taubman, ${ }^{2} \mathrm{~K}$ Shaji, ${ }^{2} \mathrm{D}$ Quinn, ${ }^{2} \mathrm{~J}$ Watson, ${ }^{2} \mathrm{I}$ Abubakar, ${ }^{1} \mathrm{~A}$ Hayward. ${ }^{1} \mathrm{UCL}$, London, UK; ${ }^{2}$ Health Protection Agency, London, UK; ${ }^{3}$ Find and Treat TB Project, London, UK

Background Radiographic screening for pulmonary tuberculosis is used in high risk groups in many countries. There are no published reports of the sensitivity and specificity of this technique in an operational setting with high screening throughput. A Department of Health screening programme has used mobile digital chest radiography to identify pulmonary tuberculosis amongst homeless people, problem drug users and prisoners in London since April 2005. We aimed to use data from this programme and from national tuberculosis surveillance to establish the sensitivity and specificity of mobile digital X-ray screening for the identification of pulmonary tuberculosis.

Methods Data collected on those screened for the period March 2005 to March 2010 were collated. Abnormal radiographs were classified as: 1) suspected active tuberculosis; 2) signs of old inactive tuberculosis; 3) other suspicious findings that warrant further investigation (eg, possible cancer); 4) abnormal but no further investigation required; 5) normal. Demographic data from those screened were matched to the national tuberculosis surveillance database using probabilistic algorithms. Duplicates within the screening database (ie, persons having been screened more than once) were removed using the same algorithms. To calculate sensitivity and specificity all those who were referred with suspected active tuberculosis were classified as 'screen positive' with all others classified as 'screen negative'. The gold standard comparator was a notification of pulmonary tuberculosis within the four months after screening. Non-pulmonary cases of tuberculosis were also excluded.

Results 39225 individuals were screened during the study period, $75 \%$ were male and the median age was 36 (IOR 27-45). 430 of those screened were referred with suspected active tuberculosis for further investigation. Matching of the screening data to surveillance data identified 44 screened individuals who were notified with pulmonary tuberculosis within 4 months of screening, 36 of these were screen positive. Based on these results, sensitivity was $82 \%$ (95\% CI 67.3 to $91.8 \%$ ) and specificity $99.3 \%$ (95\% CI 99.1 to $99.3 \%$ ) (see Abstract S6 Table 1).

Abstract S6 Table 1 Screened cases with suspected active pulmonary TB compared to gold standard (notification of pulmonary tuberculosis within 4 months of screening)

\begin{tabular}{llrrr}
\hline & & \multicolumn{2}{c}{ Gold standard } & \\
\cline { 3 - 5 } & & Positive & Negative & Total \\
\hline Screen result & Positive & 36 & 312 & 348 \\
& Negative & 8 & 38869 & 38877 \\
& Total & 44 & 39181 & 39225 \\
\hline
\end{tabular}

Conclusion High throughput mobile digital radiographic screening for pulmonary tuberculosis has high sensitivity and specificity in high risk groups and should be considered as a key tool for active case finding in these populations.

\section{Economics and the burden of respiratory disease}

\section{S7 ANNUAL TRENDS IN ASTHMA EMERGENCY ATTENDANCE,} HOSPITAL ADMISSION AND READMISSION

doi:10.1136/thx.2010.150912.7

V Kuan, S J Quantrill. Department of Respiratory Medicine, Whipps Cross University Hospital, London, UK

Introduction and Objectives Recent government initiatives rooting care of chronic conditions in primary care settings with particular emphasis on reducing hospital use require on-going collection of high-quality data. However, in most Trusts this data are not systematically reported. Data around readmissions have recently become a priority issue with proposals to penalise secondary care Trusts with high readmission rates.

Methods Asthma emergency attendances and reattendances to our Trust from 2003/4 to 2009/10 and hospital admissions and readmissions from 2001/2 to 2009/10 were obtained from Symphony and Patient Administration System (PAS). For hospital admissions discharge coding was used to identify cases.

Results 8155 emergency attendances were recorded over the 7 year period between 2003/4 and 2009/10, of which 4691 were adult ( $\geq 18$ years) and 3464 children ( $<18$ years). During this time, 2927 patients out of 4479 (65\%) attended just once. Emergency 